\title{
Le tumulus de Kervingar (Plouarzel, Finistère) : un regard nouveau sur les débuts de la recherche archéologique d'après-guerre en Bretagne
}

The Kervingar tumulus (Plouarzel, Finistère): a new look at the beginnings of archaeological research in post-war Brittany Der Grabhügel von Kervingar (Plouarzel, Finistère): Neue Betrachtungen zu den Anfängen archäologischer Forschung in der Nachkriegszeit in der Bretagne El túmulo Keringar (Plouarzel, Finistère): una nueva mirada en los inicios de la investigación arqueológica en el momento de la posguerra en Bretaña

Elías López-Romero, Marie-Yvane Daire, Patricia Mañana-Borrazás et Laurent Quesnel

\section{OpenEdition}

\section{Journals}

Édition électronique

URL : https://journals.openedition.org/rao/2973

DOI : $10.4000 /$ rao. 2973

ISBN : 978-2-7535-5014-8

ISSN : 1775-3732

Éditeur

Presses universitaires de Rennes

Édition imprimée

Date de publication : 31 décembre 2015

Pagination : 113-128

ISBN : $978-2-7535-5012-4$

ISSN : 0767-709X

Référence électronique

Elías López-Romero, Marie-Yvane Daire, Patricia Mañana-Borrazás et Laurent Quesnel, « Le tumulus de Kervingar (Plouarzel, Finistère) : un regard nouveau sur les débuts de la recherche archéologique d'après-guerre en Bretagne », Revue archéologique de l'Ouest [En ligne], 32 | 2015, mis en ligne le 28 avril 2018, consulté le 22 août 2022. URL : http://journals.openedition.org/rao/2973 ; DOI : https://doi.org/ $10.4000 /$ rao.2973 


\title{
Le tumulus de Kervingar (Plouarzel, Finistère) : un regard nouveau sur les débuts de la recherche archéologique d'après-guerre en Bretagne
}

\author{
The Kervingar Tumulus (Plouarzel, Finistère): \\ A New Look at the Beginnings of Archaeological Research in Post-war Brittany
}

\author{
Elías López-Romero ${ }^{a}$, Marie-Yvane DAIre ${ }^{\mathrm{b}}$, \\ Patricia MañAnA-BorrazÁs ${ }^{\mathrm{c}}$ et Laurent QuesneL ${ }^{\mathrm{d}}$
}

\begin{abstract}
Résumé : À partir d'une série de documents inédits, ce travail revient sur la fouille de 1953 du tumulus de l'âge du Bronze ancien de Kervingar (Plouarzel, Finistère) par Pierre-Roland Giot (1919-2002). La révision de ces documents et la discussion du contexte de l'époque nous permettent de replacer cette fouille comme l'une des pièces clés du renouveau scientifique qui s’opérera au sein de l'archéologie française dès la fin des années 1940. Elle constitue par ailleurs et avant tout l'un des travaux fondamentaux pour comprendre la pensée scientifique qui mènera à la création du « Laboratoire d'Anthropologie, Préhistoire, Protohistoire et Quaternaire armoricains » et qui marquera par la suite une grande partie de la recherche archéologique de l'Ouest de la France durant toute la seconde moitié du xxe siècle. L'existence d'un film inédit sur les travaux nous offre également l'occasion de proposer une méthodologie novatrice d'analyse d'image, avec restitution tridimensionnelle numérique d'une partie de la fouille. Cette perspective ouvre une nouvelle voie dans l'exploitation scientifique des archives anciennes.
\end{abstract}

\begin{abstract}
Drawing from a set of unpublished material, this paper revisits the 1953 excavation of the Early Bronze Age mound of Kervingar in Plouarzel (Finistère) by Pierre-Roland Giot (1919-2002). Reviewing these documents in a historical scientific context allows us to present the excavation of this site as a key example of the scientific revival occurring within French archaeology in the late 1940s. Above all, Kervingar represents a fundamental early example of the scientific rigor characteristic of the Laboratoire d'Anthropologie, Préhistoire, Protohistoire et Quaternaire armoricains, a laboratory that would come to lead most of the regional research in Western France throughout the second half of the twentieth century. Furthermore, previously unreleased footage of the fieldwork in Kervingar presents an opportunity to conduct an innovative image analysis to obtain a $3 D$ digital reconstruction of several aspects of the 1953 excavations. This method underscores the utility of scientific analyses of archival image collections.
\end{abstract}

Mots clés : tumulus, âge du Bronze ancien, historie de l'archéologie, photogrammétrie, Structure from Motion (SfM), traitement d'images, Bretagne, Finistère.

Keywords: Round barrow, Early Bronze Age, History of Archaeology, Photogrammetry, Structure from Motion (SfM), Image analysis, Brittany, Finistère.

\footnotetext{
a Department of Archaeology, Durham University, South Road, Durham DH1 3LE, UK. (lopezelias@hotmail.com)

b Laboratoire Archéosciences UMR 6566 CReAAH - Centre de Recherche en Archéologie, Archéosciences, Histoire, Bâtiment 24-25 Campus de Beaulieu, 263 Avenue du général Leclerc, Rennes, France. (marie-yvane.daire@univ-rennes1.fr)

'Institute of Heritage Sciences, Spanish National Research Council, Incipit, CSIC, San Roque 2, 15704 Santiago de Compostela, Spain. (patricia. manana-borrazas@incipit.csic.es)

' Laboratoire Archéosciences UMR 6566 CReAAH - Centre de Recherche en Archéologie, Archéosciences, Histoire, Bâtiment 24-25 Campus de Beaulieu, 263 Avenue du général Leclerc, Rennes, France. (laurent.quesnel@univ-rennes1.fr)
} 


\section{INTRODUCTION}

En septembre 1953 une équipe dirigée par Pierre-Roland Giot (1919-2002) entreprend, en compagnie du D vétérinaire Louis L'Hostis (1897-1971), la fouille du tumulus de l'âge du Bronze ancien de Kervingar, dans la commune de Plouarzel (Finistère; fig. 1). La publication des travaux (Giot, 1954; Milon et Giot, 1954; Giot, 1956), rend compte des découvertes faites dans les trois chambres funéraires trouvées au sein du monument mais surtout de la structure générale du tumulus, de sa stratigraphie précise et des méthodes de fouille et d'enregistrement des données. Peu ou très sommairement citée dans les travaux postérieurs sur les recherches archéologiques et préhistoriques de l'ouest, cette fouille constitue cependant une pièce clé dans la compréhension du renouveau scientifique qui va se produire en France pendant les deux décennies suivant la fin de la seconde guerre mondiale.

Les recherches menées dans le cadre du projet ICARE (Iconographie et Collections d'Anthropologie de REnnes; Daire et López-Romero, 2011; Daire et López-Romero, 2012; López-Romero et Daire, 2013; Daire et al., 2013; Rocher, 2013) sur les archives de l'ancien laboratoire d'anthropologie, préhistoire, protohistoire et Quaternaire armoricains, crée par Giot, ont permis de redécouvrir toute une série d'enregistrements réalisés par lui entre 1952 et vers 1970, dont un court-métrage de douze minutes sur le site de Kervingar. D'autres documents restés inédits (quelques notes, courriers, photographies et dessins) sur les travaux ont également été trouvés parmi les archives du laboratoire.

Parfois négligées par la recherche actuelle en tant que source d'information primaire, les archives iconographiques des pionniers de l'archéologie n'attirent pour l'essentiel que les historiens de la discipline et les quelques intrépides archéologues qui osent parfois plonger dans des dossiers le plus souvent poussiéreux pour mieux appréhender l'historique des travaux sur un site en particulier. Au-delà de quelques renseignements sur la présence de tel ou tel élément archéologique au moment de la fouille, de l'altération des éléments aujourd'hui conservés ou de la localisation plus ou moins précise de la zone où eurent lieu les travaux anciens, les limites de ce type de source documentaire sont en général nombreuses et ne permettent pas a priori une utilisation aisée des informations scientifiques qu'il contient.

Dans cet article, nous revenons sur la fouille de Kervingar avec un double objectif. Premièrement, restituer le rôle de " pierre maitresse " que la fouille de ce tumulus acquiert dans la construction d'une archéologie scientifique d'après-guerre, et notamment dans la préfiguration d'une archéologie pluridisciplinaire qui trouvera, dans le laboratoire d'anthropolo-
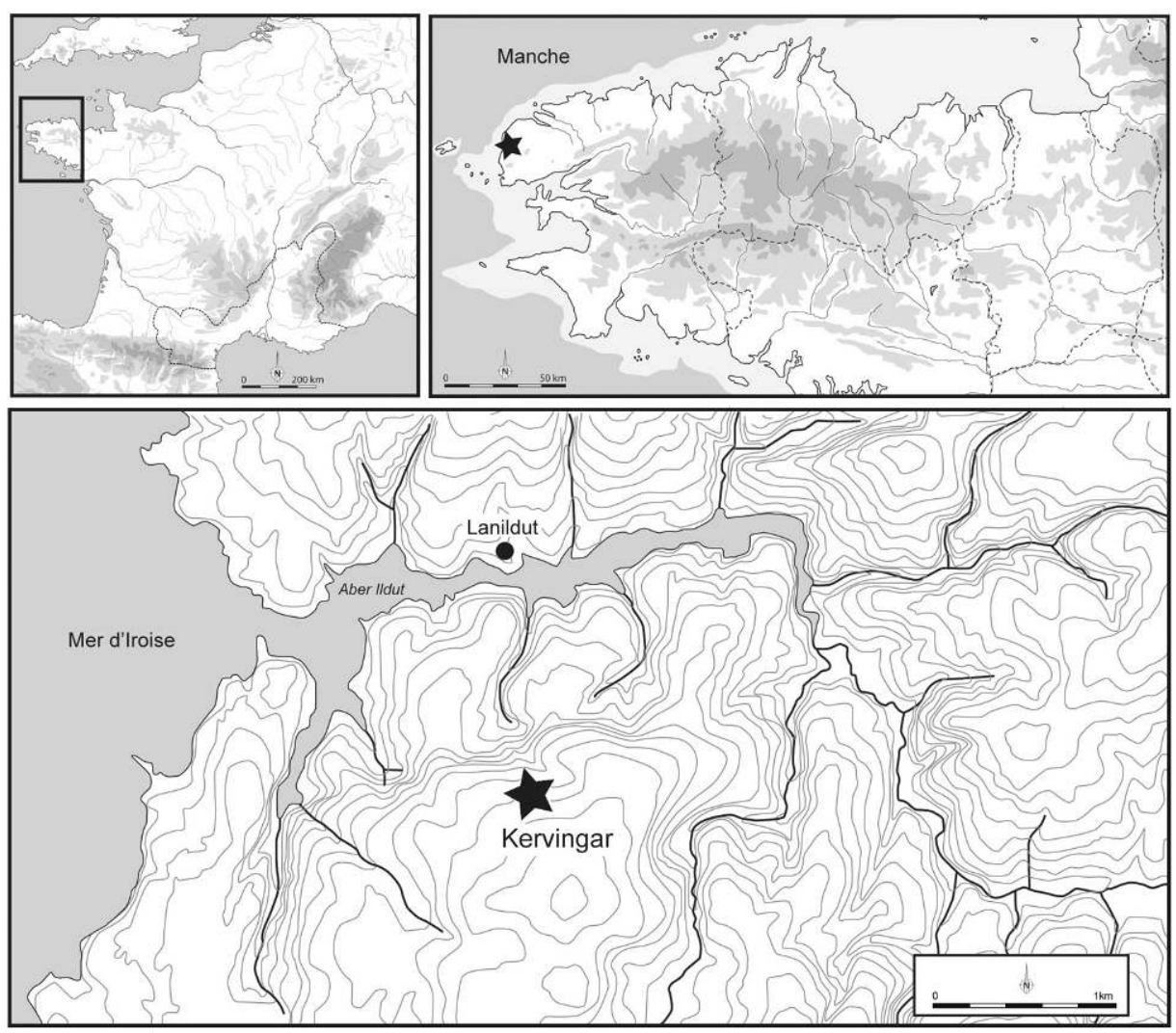

Figure 1 : Carte de localisation du tumulus de Kervingar. Plouarzel, Finistère; DAO L. Quesnel.

Figure 1: Location of the Kervingar mound. 
gie de la faculté de sciences de Rennes, l'un de ses premiers et plus représentatifs points de référence. Deuxièmement, restituer la valeur, en tant que source scientifique de premier ordre, des archives iconographiques anciennes à travers une perspective novatrice qui, grâce à l'utilisation de nouvelles technologies actuelles, permet la restitution métrique de plusieurs éléments de la fouille à partir d'un ensemble d'images.

\section{KerVingar, 1953}

À partir de la période d'entre-deux-guerres, un renouveau, aussi bien générationnel que scientifique, s'opère autour des activités archéologiques. L'idée d'une réglementation des fouilles - face à une importante liberté d'action individuelle qui avait pour l'essentiel caractérisé la fin du XIX ${ }^{e}$ siècle et les premières décennies du $\mathrm{Xx}^{\mathrm{e}}$ siècle - se consolide peu à peu. L'importance des sites en tant qu'entités dans un contexte spatial et territorial large, et en tant qu'entités interdépendantes, explicatives et complémentaires à l'objet archéologique, gagne progressivement de la valeur. Lintérêt pour le développement de nouvelles techniques de fouilles adaptées à cette conception changeante devient de plus en plus important (Hurel, 2007, p. 244-249).

Dans ce contexte, la promulgation, en 1941, de la loi réglementant les fouilles archéologiques (" loi Carcopino" du 27 septembre ${ }^{1}$ ) et, quatre ans plus tard, du décret portant sur la création des circonscriptions des antiquités préhistoriques et historiques ${ }^{2}$, sert à consolider un contrôle plus effectif des activités de terrain ainsi que des objets mis au jour de façon fortuite ou comme résultant de ces activités.

Héritier de l'institut finistérien d'études préhistoriques dont il est jeune membre dès 1937 puis « conservateur » du musée de Penmarc'h, siège de l'institut, depuis 1947 (LópezRomero et Daire, 2013) mais aussi fonctionnaire d'État (en tant que membre du CNRS depuis 1943 et directeur de la $I^{e}$ circonscription des antiquités préhistoriques dès 1948), Pierre-Roland Giot se trouve à la fin des années 1940 à cheval sur deux conceptions antagonistes de la pratique archéologique, désormais nettement séparées par la loi de 1941 : celle d'une archéologie traditionnelle fondée sur les principes de la liberté d'action individuelle (réalisée toutefois le plus souvent au sein d'une société savante) et celle d'une archéologie règlementée et supervisée par l'administration centrale.

Malgré cette position, on peut dire, sans risque d'erreur, que la suprématie de la science officielle sur le droit individuel se trouve à la base des travaux de Giot dès le début de

1. Loi du 27 septembre 1941, Journal Officiel de l'État Français du 15 octobre 1941, sur la réglementation des fouilles archéologiques.

2. Décret $n^{\circ}$ 45-2098 du 13 septembre 1945, Journal officiel de la République française. sa carrière, constituant même le leit-motiv de son activité, ce principe qu'il qualifie de « recherche désintéressée » dès 1948 (Giot, 1948). Cette motivation devient également la clé qui explique une certaine obsession de Giot à faire connaître la mise en place d'une méthodologie scientifique moderne dans ses travaux dès la fin des années 1940 : «Puissent tous les préhistoriens et amateurs d'archéologie être persuadés qu'à notre époque, il est impensable d'explorer un tumulus intact ou un monument construit similaire, sans employer des techniques aussi poussées et aussi exhaustives. Agir autrement est tout simplement criminel. " (Giot, 1956, p. 102. Pour une critique de l'approche scientifiste de Giot et de la loi de 1941 voir Gouletquer, 2009.)

Malgré les efforts de fouille et de restauration réalisés depuis la fin du XIx ${ }^{e}$ siècle dans de nombreux monuments datant du Néolithique et de l'âge du Bronze, la compréhension des architectures orthostatiques et tumulaires en tant qu'ensembles complexes (aussi bien sur le plan architectural que chronologique) manquait encore d'une approche méthodologique solide et spécifique. Certains travaux réalisés dans les monuments mégalithiques du Sud-Ouest de la France (Laplace-Jauretche, 1951) avaient introduit progressivement des systèmes d'enregistrement plus complets que les méthodes traditionnelles et qui amélioraient, par exemple par l'application systématique des coordonnées cartésiennes (Laplace-Jauretche et Méroc, 1954), la qualité des informations récupérées.

Le travail qui aurait pu anticiper de plus d'une décennie ce type d'approche, celui de la fouille en juin 1939 du tumulus de La Motta à Servel (Lannion, Côtes-d'Armor) par Albert Van Giffen (1884-1973), resta inédit jusqu’à sa publication en 1974, un an après la mort de l'archéologue néerlandais (Butler et Waterbolk, 1974). L'existence, à l'étranger, de méthodologies de fouille similaires (Grimes 1938) n'est pas mentionnée par Giot. Il en est de même pour certains travaux réalisés en Bretagne au XIx ${ }^{\mathrm{e}}$ siècle, tels que ceux du tumulus de Lothéa à Quimperlé (Nicolas et al., 2013) et du tumulus de Cruguel à Guidel (Le Pontois, 1890).

Pour ce qui est du cadre chrono-typologique, la publication, en 1951, d'une synthèse sur les tumulus de l'âge du Bronze en Bretagne en fournit une vision actualisée pour l'époque, avec la distinction de deux "séries " : une " première série " qui regroupe des tumulus généralement de grande taille avec présence d'éléments en bronze, pointes de flèche en silex et quelques objets précieux; une « seconde série " avec des éléments céramiques et une moindre présence d'armes en bronze (Cogné et Giot, 1951). Ce cadre resta problématique et sera revu par la suite (Briard et al., 2001; Gabillot, 2010).

Connaissant et appréciant les travaux de Van Giffen, Giot va mettre en pratique une méthodologie de terrain similaire 
à celle de ce dernier, et il va l'appliquer pour la première fois dans la région à la fouille de Kervingar. Bien au-delà du simple alignement avec la perspective de terrain du précédent, Giot présente explicitement cette fouille comme étant l'exemple d'une méthodologie moderne qui devra marquer les travaux archéologiques dans la région par la suite. Ce jugement est-il juste? En quoi la fouille de Kervingar représente-t-elle un tournant dans la façon d'aborder les problématiques théoriques et méthodologiques de terrain?

La formation scientifique initiale de Giot, plus particulièrement en sciences et géologie, à Paris puis à Grenoble, constitue un premier élément de réflexion a cet égard, ainsi que son travail de thèse doctorale, initié en 1945 et soutenu à Rennes en 1950, qui portait sur l'anthropologie des populations bretonnes de la préhistoire à son époque (Giot, 1951). C'est donc sans surprise que, dans ses fouilles et travaux, on retrouve systématiquement l'héritage scientifique d'une vision fortement basée sur les sciences de la nature. Cette caractéristique de la formation initiale de Giot jouera, par la suite, un rôle essentiel sur le plan épistémologique, puisqu'elle va doter la recherche archéologique régionale d'après-guerre d'un contexte ouvertement objectiviste, empiriste et "scientifique " (c'est-à-dire néo-positiviste) domaine où elle avait été jusqu'alors largement déficitaire.

La mise en place d'une telle méthodologie doit être contextualisée dans l'expérience progressivement acquise par Giot lors de fouilles préalables dans le Finistère, telles celles des tumulus de Beg an Dorchenn ("Pointe de la Torche ») à Plomeur (Giot, 1947; Giot et Guilcher, 1947) ou du bourg de Kersaint-Plabennec (Giot et Cogné, 1948), ainsi que dans d'autres actions suite à des découvertes fortuites (Giot, 1953a). C'est cependant dans la fouille de Kervingar que ce positionnement semble se matérialiser, pour la première fois de façon intégrée, dans des choix méthodologiques précis.

Une première décision en ce sens fut la réalisation d'une topographie détaillée du terrain par «nivellement à la planchette et à la règle à éclimètre, à tous les mètres au centre, puis tous les deux mètres » qui prit tout une journée de travail (Giot, inédit a). Suit la délimitation des quadrants de la fouille, avec le tracé de deux axes nord-sud et est-ouest. Puisque toute fouille constitue un exercice de destruction, et suivant la perspective de Van Giffen, Giot procède par " quadrants et ponts-témoins opposés par le sommet " à partir des deux axes mentionnés (Giot, 1956); ces pontstémoins, larges de $0,75 \mathrm{~cm}$, viendront à l'appui de l'étude des relations stratigraphiques au sein du tumulus tout au long des travaux de fouille (fig. 2 et 3). L'intention ultime de cette planification est de permettre le repérage exact des éléments qui se trouvent sous le niveau du sol contemporain, par une fouille intégrale du monument. C'est uniquement par le biais d'une fouille de ce type que l'on pourra, d'après Giot, avancer sur les plans qualitatif et quantitatif dans la connaissance des architectures tumulaires monumentales. L'importance accordée à la fouille intégrale repose sur une réflexion de base qui, à la différence de la plupart des approches précédentes du monumentalisme régional, présente le site archéologique comme une entité complexe dont les parties constituantes (masses tumulaires, chambres

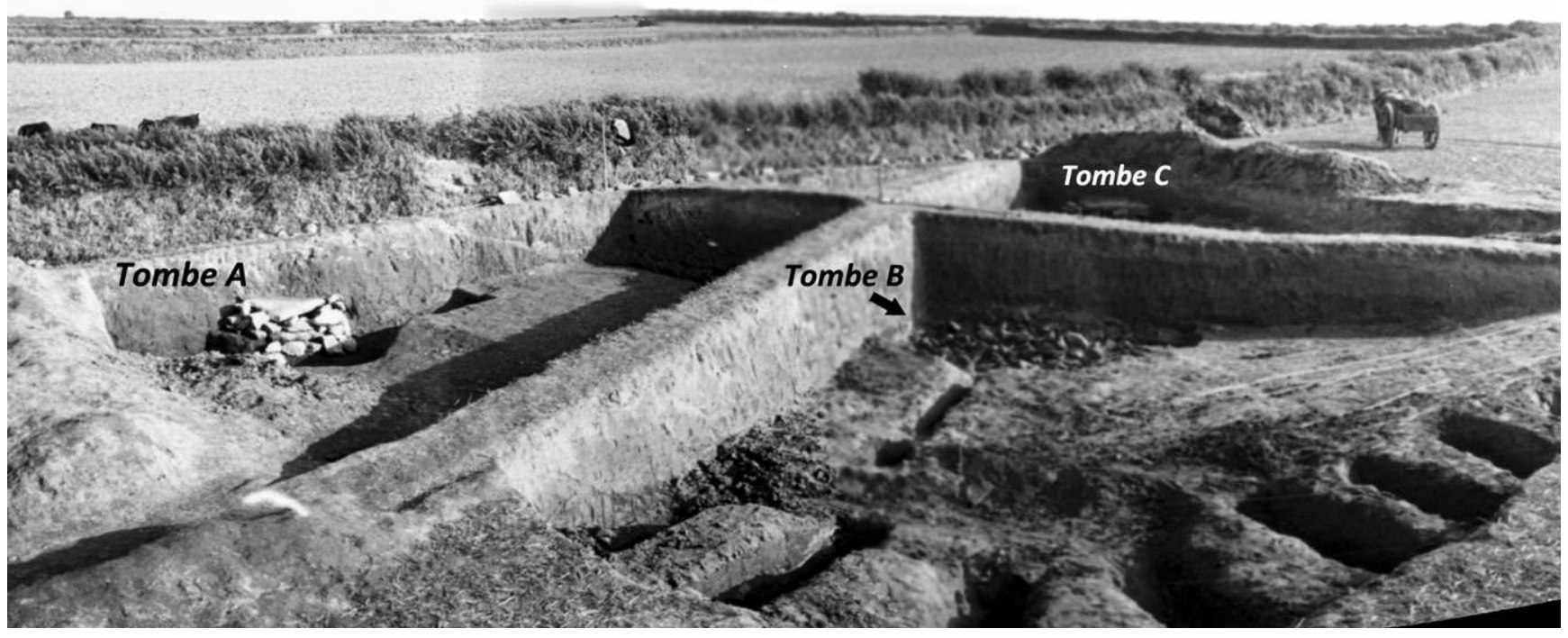

Figure 2 : Vue panoramique prise du nord-ouest du tumulus de Kervingar au moment de l'achèvement des travaux, le 26 septembre 1953. Avec localisation des tombes $A, B$ et $C$. Cliché P.-R. Giot.

Figure 2: Panoramic view from the north-west of the Kervingar excavation, 26 ${ }^{\text {th }}$ September 1953, showing Grave $A, B$ et $C$. 
Figure 3 : Plan de la fouille du tumulus de Kervingar. Modifié d'après Giot, 1956. DAO E. López-Romero.

Figure 3: Excavation plan of the Kervingar mound.

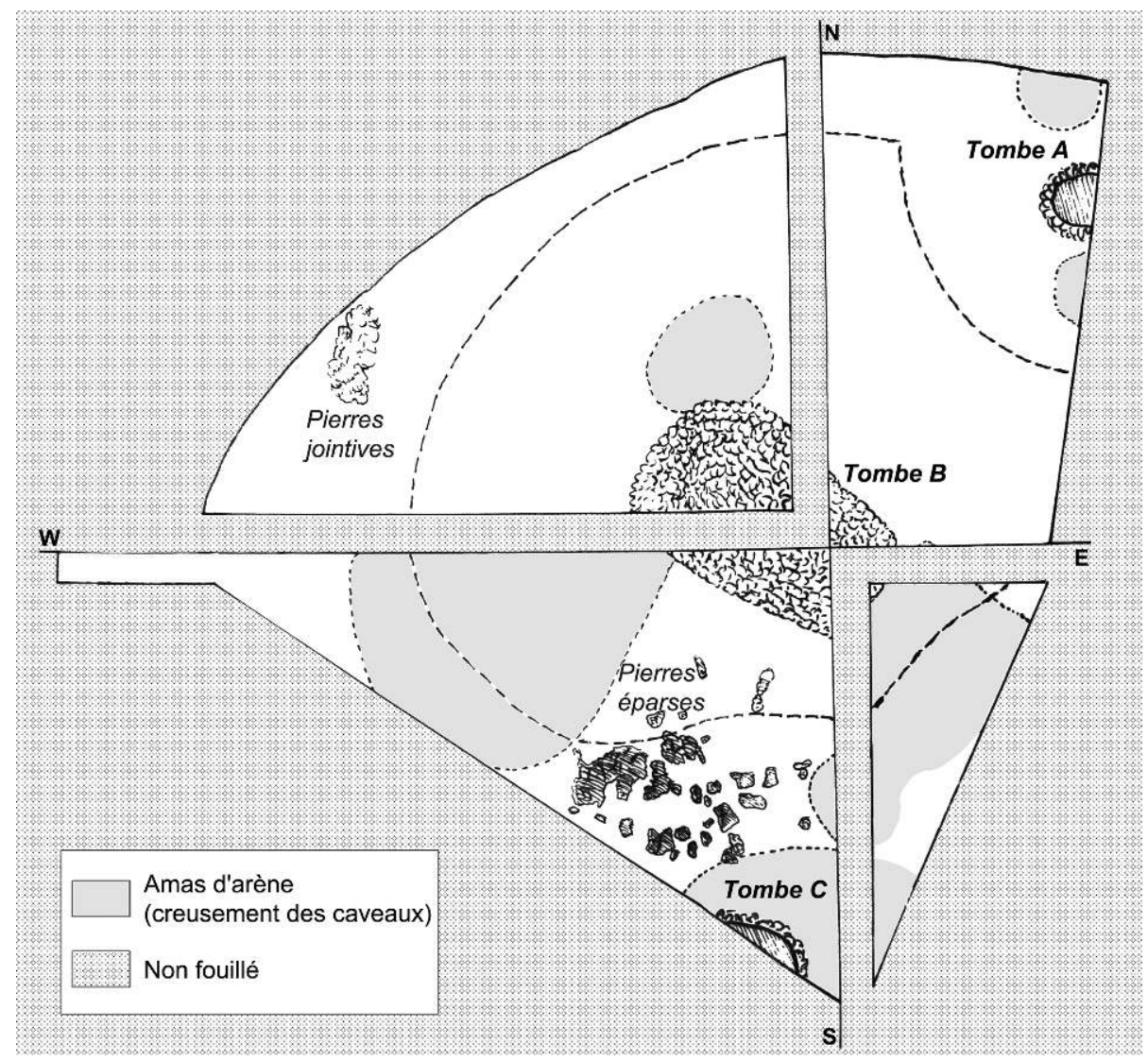

funéraires et relations spatiales entre les différents éléments trouvés à l'intérieur des chambres) sont interdépendantes et nécessitent d'être analysées avec une égale intensité (Giot, 1953b). C'est cette perspective qui permettra à Giot d'établir un ordre chronologique relatif pour la construction des structures trouvées lors des travaux à Kervingar : construction des " tombes accessoires ${ }^{3}$ " $\mathrm{A}$ et $\mathrm{C}$ (chacune avec un tumulus), construction de la «tombe centrale » B (avec recouvrement partiel de certaines parties des tumulus A et $\mathrm{C}$ ), construction d'un lit de pierres jointives en guise de cairn dans le secteur nord-ouest, puis construction d'un grand tumulus qui recouvre l'ensemble des structures. On ne saurait trop souligner l'importance de cette perspective, surtout en ce qui concerne la valeur accordée aux masses tumulaires dont l'étude détaillée allait tarder à être instaurée dans certaines régions de la façade atlantique européenne.

Paradoxalement, la qualité du plan général publié de la fouille est pauvre. Des dessins des chambres, d'une meilleure qualité, ont également été réalisés mais ils étaient restés inédits jusqu'à présent (fig. 4 à 6).

Parallèlement à l'enregistrement exhaustif des éléments présents dans la fouille et à ses relations stratigraphiques

3. Nous reprenons ici la dénomination attribuée par Giot aux différentes tombes; cette dénomination ne correspond en réalité qu'à leur position spatiale par rapport à la morphologie générale du monument. (horizontales et verticales; fig. 7), le travail réalisé à Kervingar se caractérise également par une adaptation aux questions scientifiques qui se posent sur le terrain, voire une anticipation de celles-ci. Même si le recours aux analyses chimiques de certains matériaux liés aux monuments n'est pas nouveau (des analyses de ce genre ont été réalisées dès la moitié du siècle précédent; Damour, 1864), l'échantillonnage systématique au moment de la fouille, comme une sorte de " réflexe " de géologue, constitue l'un des points clés de la procédure de Giot. Ce souci de documentation est en consonance avec la prise de conscience du caractère destructif des travaux (cf. supra). Il ne s'agit donc pas d'étudier seulement ponctuellement tel ou tel élément, mais d'anticiper le type et la quantité des échantillons nécessaires pour la compréhension du site et de son contexte une fois le chantier fermé. Ayant pour la première fois accès à un espace funéraire intact, et malgré la conservation différentielle du contenu des différentes chambres, Giot prélève à Kervingar une grande quantité de matière organique correspondant à l'ancien plancher de bois de la " tombe centrale » B. Cet échantillonnage constitue probablement le premier destiné à dater un monument funéraire ${ }^{4}$, et précède de plusieurs mois

4. Une lettre datée du 14 novembre 1953 rend compte d'un premier contact de P.-R. Giot avec H. L. Movius (Peabody Museum, États-Unis) sur la possibilité d'obtenir une datation pour Kervingar. 

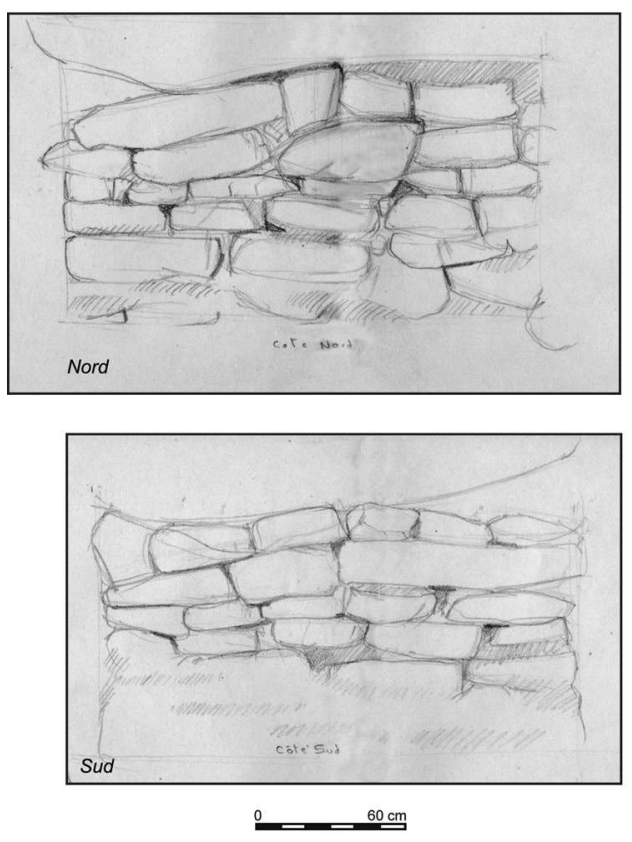

E
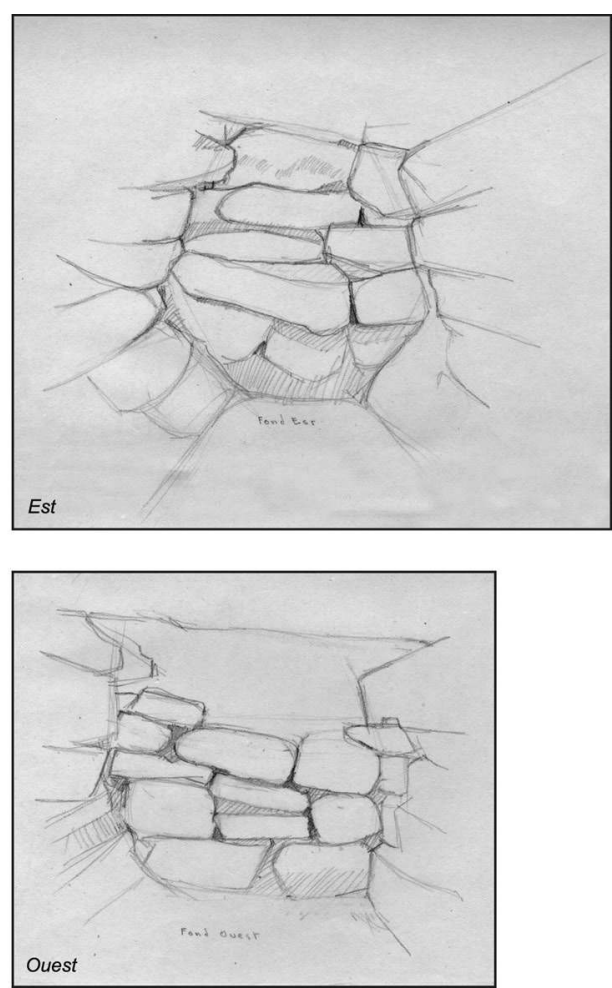

Figure 4 : Intérieur de la tombe A, d'après croquis originaux de la fouille (Archives du Centre de Documentation de l'UMR 6566 CReAAH). DAO L. Quesnel. Figure 4: View of the inside of grave $A$, from the original sketches of the excavation.
Figure 5 : Intérieur de la tombe $\mathrm{B}$, d'après croquis originaux de la fouille. Archives du Centre de Documentation de l'UMR 6566 CReAAH. DAO L. Quesnel.

Figure 5: View of the inside of grave $B$, from the original sketches of the excavation.
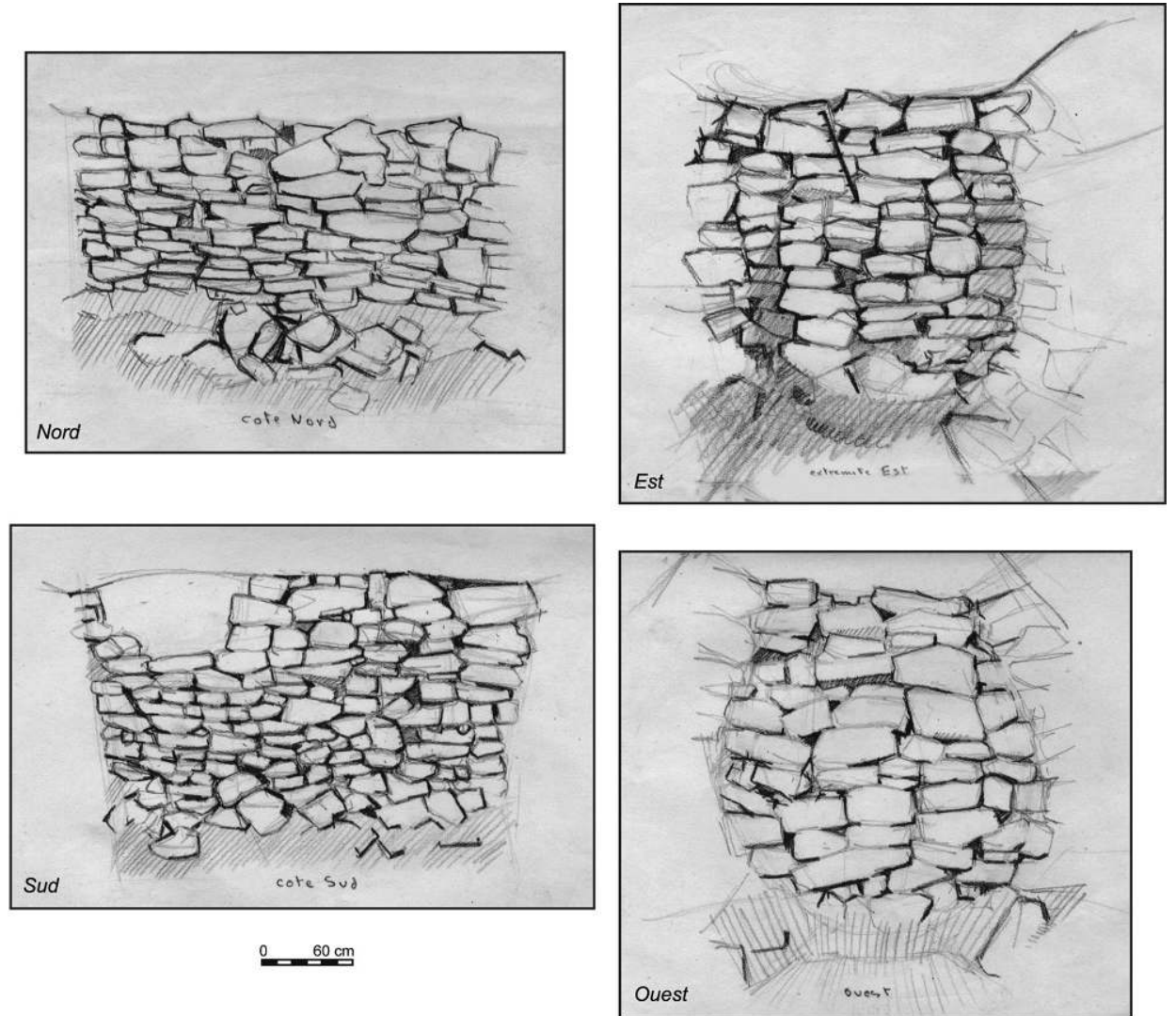

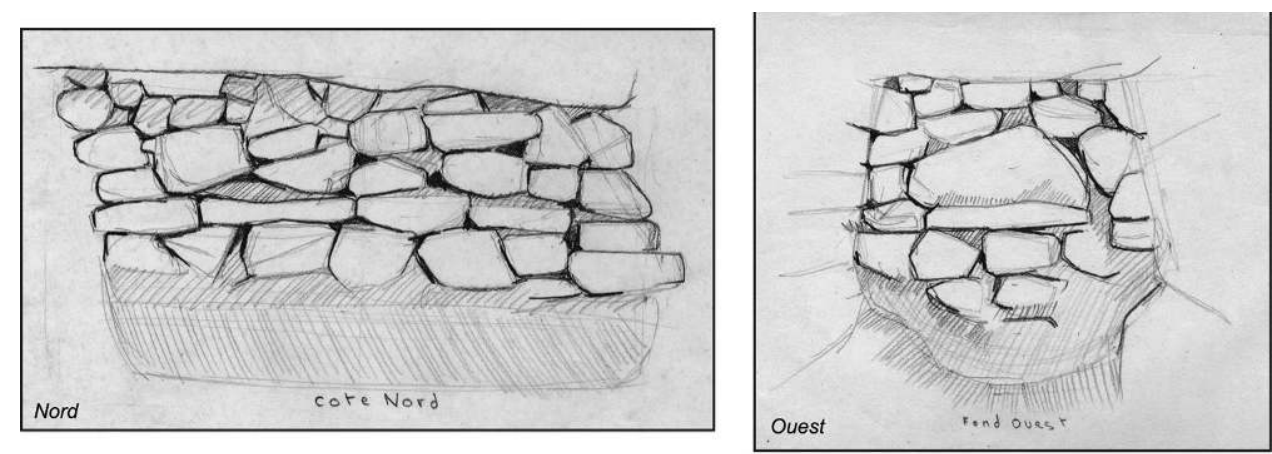

Figure 6 : Intérieur de la tombe C, d'après croquis originaux de la fouille. Archives du Centre de Documentation de l'UMR 6566 CReAAH. DAO L. Quesnel. Figure 6: View of the inside of grave $C$, from the original sketches of the excavation.
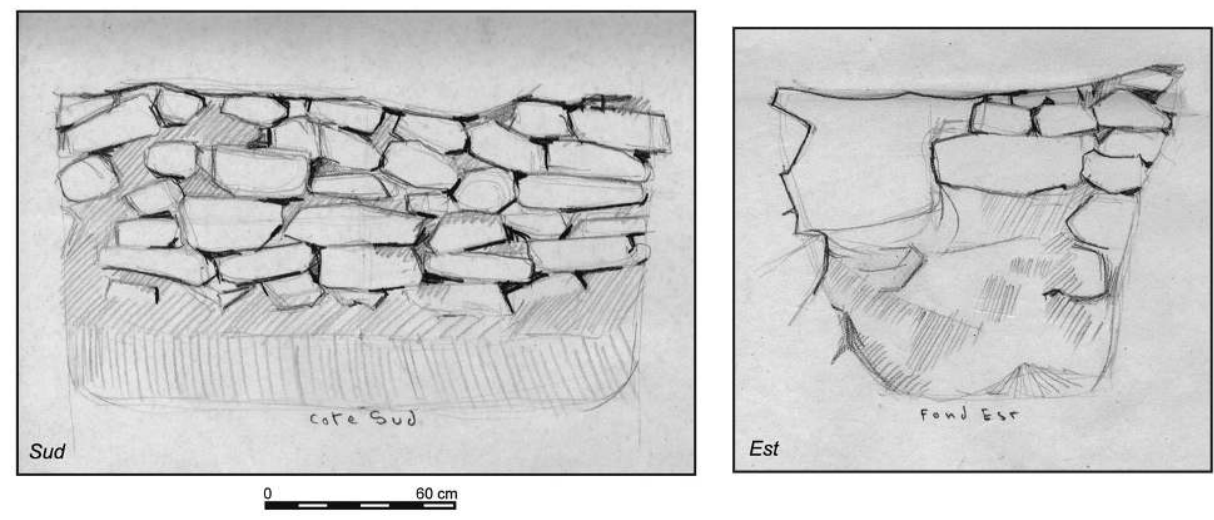

celui réalisé sur le monument de l'île Carn (Ploudalmézeau, Finistère) qui fournira, par la suite, la première date radiocarbone obtenue en Europe pour une sépulture mégalithique (GrN-1968, $5230 \pm 75$ B.P.; Giot, 1967 p. 150). Ces restes organiques de Kervingar vont donc non seulement lui permettre d'obtenir l'une des toutes premières datations absolues pour la région auprès du laboratoire de Groningen (GrN-1670, $3550 \pm$ 50; Giot, 1958; Vogel et Waterbolk, 1963) mais aussi, à un moment où la méthode du radiocarbone ne fait que ses premiers pas, de tester la fiabilité des différentes techniques instrumentales disponibles en envoyant certaines quantités du même échantillon au laboratoire de Gif-sur-Yvette (FG11a, $2934 \pm 200$ B.P.; FG11b, $3075 \pm$ 200 B.P.; Giot, 1960, p. 41-42).

Le souci de précision chez Giot est lié, comme on l'a évoqué précédemment, à la notion de complexité du site archéologique et à la prise de conscience que la fouille constitue une activité essentiellement destructrice. Dans le but de garder le maximum de documents sur l'intervention, Giot se servira de près de six mètres de pellicule photographique $35 \mathrm{~mm}$ (dont nous n'avons malheureusement pas pu retrouver la plupart des négatifs et/ou positifs; Giot, inédit b), mais aussi d'une autre méthode d'enregistrement : la réalisation d'un film sur les travaux. Par les documents comptables conservés à Rennes, nous savons que Giot avait acheté, le 17 septembre 1952, une caméra "Pathé Webo Super 16 » avec trois types d'objectifs $(1.8 / 20 \mathrm{~mm} ; 1.9 / 28 \mathrm{~mm} ; 3.5 / 50 \mathrm{~mm})$. Les nombreux enregistrements réalisés par Giot pendant près de deux décennies avec cet appareil (1952 à environ 1970) ont fait l'objet d'une restauration et d'une numérisation pour garantir sa préservation. Bien que l'analyse exhaustive de l'ensemble de ces documents ne soit pas finie et en fonction des éléments d'analyse dont nous disposons actuellement, nous travaillons sur l'hypothèse que l'enregistrement de Kervingar constitue le premier film scientifique réalisé par Giot dans le cadre de ses activités professionnelles. Ce tournage aurait probablement été précédé de plusieurs tests de l'appareil, qui correspondraient à une partie des documents à thématiques ethnographique et anthropologique qui se trouvent également parmi les films conservés.

D'autres travaux pionniers, tels que ceux des Péquart à Téviec, Hoëdic ou Er Lannic, s'étaient servis de ce mode d'enregistrement auparavant (Collectif, 2007). Le film sur Kervingar n'en reste pas moins un document d'exception. Il nous a également permis d'en extraire des informations et produits scientifiques nouveaux grâce à une reconstitution métrique tridimensionnelle à partir d'images de certains éléments de la fouille (cf. infra). Grâce à des notes inédites, nous avons eu des précisions sur le contexte et les conditions particulières de l'enregistrement de ces images : «[...] la malchance a voulu qu'étant donné que j'étais seul à pouvoir faire les travaux délicats lors de tous les moments psychologiques des événements [...] il m'était impossible de tenir la caméra, et j’ai été obligé de la confier à quelqu'un 


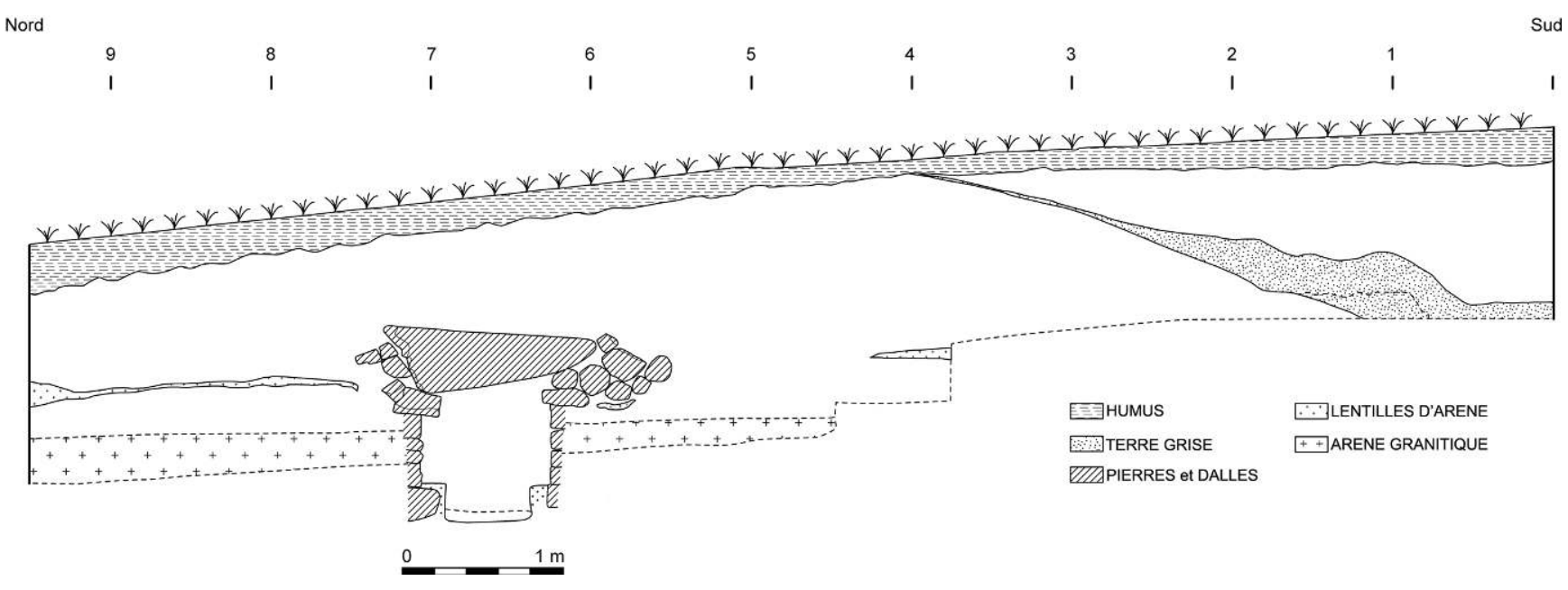

Figure 7 : Coupe N-S inédite du quadrant N-E de la fouille du tumulus de Kervingar, montrant la tombe A. DAO L. Quesnel à partir des dessins de terrain originaux. Les coupes cardinales du tumulus furent publiées dans Giot 1956.

Figure 7: North-South stratigraphy in the northeast quadrant, showing grave A. The stratigraphy of the main two axis of the mound were published in Giot 1956.

qui n'était pas du tout préparé à cet exercice, et qui a réussi à accumuler toutes les fautes techniques qu'il ne fallait pas faire, en particulier de bouger continuellement tout en filmant. Surchargé de travaux divers, je n'ai pas actuellement le temps matériel de monter le film et faire les coupures indispensables » (Giot, inédit b p. 9). Le film de Kervingar est donc tombé dans l'oubli.

\section{UN REGARD NOUVEAU : RESTITUTION PAR L'IMAGE DE LA FOUILLE DE KERVINGAR}

Depuis quelques années maintenant, plusieurs projets d'enregistrement tridimensionnels du patrimoine ont été lancés. Les objectifs sont nombreux : préservation sous forme numérique d'éléments susceptibles de disparaître, représentation détaillée du patrimoine, monitorisation de structures et bâtiments, manipulation virtuelle d'architectures et d'objets de grande taille ou très fragiles, création de reconstructions virtuelles, élaboration de présentations didactiques pour le grand public, etc.

Ce mouvement a été largement favorisé par une croissante disponibilité et simplification des méthodes permettant l'obtention de ce type de produits, parmi elles certaines technologies laser (LiDAR-TLS) et photogrammétriques. La plupart des projets finalisés ou en cours concernent l'analyse des architectures (Gillings, 2000), des peintures et gravures rupestres (Simpson et al., 2004), des objets et collections des musées (Beraldin et al., 2000) ou encore des structures archéologiques en cours de fouille (Doneus et al., 2011). D’autres perspectives récentes ont intégré ce type de démarche aux analyses de l'érosion des sites (López-Romero et al., 2014). Tous ces projets partent de deux principes fondamentaux : celui de l'accès direct à l'élément que l'on veut documenter et celui d'une planification méthodique de l'enregistrement à réaliser.

L'exploitation scientifique des archives iconographiques anciennes est par contre restée en marge de ce développement, avec tout de même quelques expériences ponctuelles sur l'utilisation de photographies anciennes pour la restitution volumétrique d'architectures disparues (Loannidis et al., 1996; Grün et al., 2004). Connaissant l'existence et le potentiel de ces méthodes, nous nous sommes posé la question de savoir si une restitution tridimensionnelle et, ce qui est plus important, métrique, d'objets et d'architectures permettant la récupération d'informations scientifiques nouvelles, pouvait être obtenue à partir de collections iconographiques très anciennes qui ne remplissent en général aucun des deux principes mentionnés ci-dessus. C'est-à-dire, qu'ils ne nous permettent pas de revenir sur l'élément ou l'objet étudié (car il a été depuis détruit, reste inaccessible ou concerne un aspect qui ne peut être reproduit à nouveau - cas de l'étude des méthodologies d'une fouille ancienne) et dont les conditions d'obtention/capture/enregistrement n'avaient jamais été prévues pour atteindre une telle restitution (prise d'images non planifiée, série d'images peu nombreuses, absence d'une couverture totale de la superficie de l'élément étudié, images floues ou de qualité médiocre, absence d'information sur la calibration et les caractéristiques des appareils, etc.). De même, il nous semblait intéressant de savoir si cette restitution pouvait être obtenue à partir des différents types de supports présents dans la col- 
lection de l'ancien laboratoire d'anthropologie, préhistoire, protohistoire et Quaternaire armoricains (positifs sur papier, négatifs sur plaque de verre, négatifs sur film, films $16 \mathrm{~mm}$, etc.).

Au-delà des résultats très positifs obtenus à partir d'images datant de 1911/1912 (López-Romero, 2014), l’analyse du court-métrage de Kervingar nous a permis de répondre à ces questions dans des conditions extrêmes (dues aux caractéristiques de l'enregistrement et du support) et de contribuer, de ce fait, à la connaissance des procédures de terrain mises en place en ces débuts de la reprise des recherches archéologiques d'après-guerre.

Pour ce faire, nous avons utilisé les principes de la photogrammétrie d'un objet proche (Close-Range Photogrammetry), qui permet d'obtenir un levé tridimensionnel et métrique de détail des éléments à enregistrer. Parmi les logiciels qui permettent l'application de cette méthode nous avons utilisé Agisoft PhotoScan (C) qui consiste à appliquer la technique structure from motion (SfM; Agarwal et al., 2011; Verhoeven, 2011), largement utilisée en archéologie et patrimoine depuis quelques années maintenant. Cette technique développée principalement dans le domaine de la vision computationnelle (computer vision) est basée sur le principe de la capacité physiologique humaine à reconnaître la forme et la structure d'une scène à travers la vision et le mouvement autour de cette scène. Dans la pratique, elle facilite énormément l'obtention de modèles 3D puisqu'elle permet la restitution tridimensionnelle à partir d'images acquises depuis différents points de vue même en absence de paramètres de calibration ou toute autre information sur l'appareil. SfM a été utilisé dans des projets tels que Photosynth [http://photosynth.net], Photo Tourism [http://phototour.cs.washington. edu/ ou encore Building Rome in a Day (Agarwal et al., 2011) qui se servent d'images provenant de sources différentes et partagées en ligne.

Avec PhotoScan (C), à partir d'une série d'images non calibrées, il est possible de calculer la position de la caméra et l'alignement des clichés au moyen de toute une série de procédures et de réglages qui mènent, à terme, à une triangulation photogrammétrique automatique. Cette triangulation reconstruit un nuage de points $3 \mathrm{D}$ à partir duquel les superficies tridimensionnelles vont être créées. Comme il a été signalé, pour obtenir un résultat de précision, l'acquisition d'images de qualité et la présence de points de référence métrique (qui seront le plus souvent mesurés à l'aide d'un théodolite laser) sont essentielles. Sous ces conditions il est possible d'obtenir des modèles d'une précision de l'ordre du millimètre. En résulte une représentation avec des valeurs métriques tridimensionnelles qui peut être figurée de façon bidimensionnelle (ortho image) ou tridimensionnelle (modèle numérique tridimensionnel).
Ces procédures analytiques ont dû être adaptées aux limites imposées par le court-métrage de Kervingar.

Premièrement, le fait que la quasi-totalité du courtmétrage ait été réalisée avec l'appareil tenu à la main a eu pour conséquence que de nombreuses images (photogrammes) sont très floues (mouvements rapides et instabilité de l'appareil, dont la cause nous est désormais connue par les notes inédites de Giot citées plus haut). Le choix a donc été pris d'extraire image par image celles qui, pour un élément ou section de la fouille donné, présentaient une meilleure qualité visuelle. À partir de la version numérisée du film original $(16 \mathrm{~mm})$, l'extraction des images a été réalisée à l'aide du logiciel « open source "Video LAN VLC Media Player; les images sélectionnées ont été enregistrées sous format PNG (Portable Network Graphics, format qui préserve l'intégralité des données d'origine lors de la compression des archives). Chaque image traitée a une dimension finale de 768 x 576 pixels pour une taille d'archive très basse qui varie entre $441 \mathrm{~KB}$ et $661 \mathrm{~KB}$. Elles ont été ensuite intégrées dans PhotoScan (C) pour leur alignement et, finalement, pour la restitution des modèles tridimensionnels.

Un deuxième problème concerne la couverture de chaque élément d'intérêt dans la fouille, qui a pu être faite lors de l'enregistrement en 1953. D'un côté, il n'a pas été possible de restituer la totalité des structures visibles dans le tournage, à cause du nombre insuffisant d'images permettant la création des modèles numériques. D'un autre côté, la précision et la qualité des résultats ont été très conditionnées par la position et le mouvement du cameraman par rapport au sujet enregistré; contrairement au mouvement enveloppant nécessaire pour une restitution photogrammétrique correcte par SFM, les vues frontales et les vues depuis un même point prédominent dans le court-métrage de Kervingar, ce qui donne comme résultat des perspectives coniques.

Troisièmement, en l'absence de points de repère fixes à coordonnées connues, l'information sur l'échelle, pour la restitution métrique des modèles, doit être fixée en coordonnées relatives, ayant été récupérée à partir des jalons gradués (de $60 \mathrm{~cm}$ de longueur) visibles sur les images. Après mise à l'échelle, les modèles tridimensionnels crées dans PhotoScan (C) ont été exportés comme archives PLY (Stanford Polygon File Format) vers le logiciel MeshLab (C) pour nettoyage et pour translation du système de coordonnées. Un système orthogonal arbitraire $\mathrm{X}, \mathrm{Y}, \mathrm{Z}$ a été appliqué à chaque modèle. Finalement, un rendu photo-réaliste a été obtenu par nappage à partir des images source (fig. 8).

Trois modèles permettant une analyse numérique tridimensionnelle ont ainsi pu être obtenus : deux pour la " tombe " A et un pour la «tombe " $\mathrm{C}$ (tabl. 1). La pauvre qualité des séries d'images correspondants à deux moments de la fouille de la «tombe centrale " B n'a pas permis l'ob- 


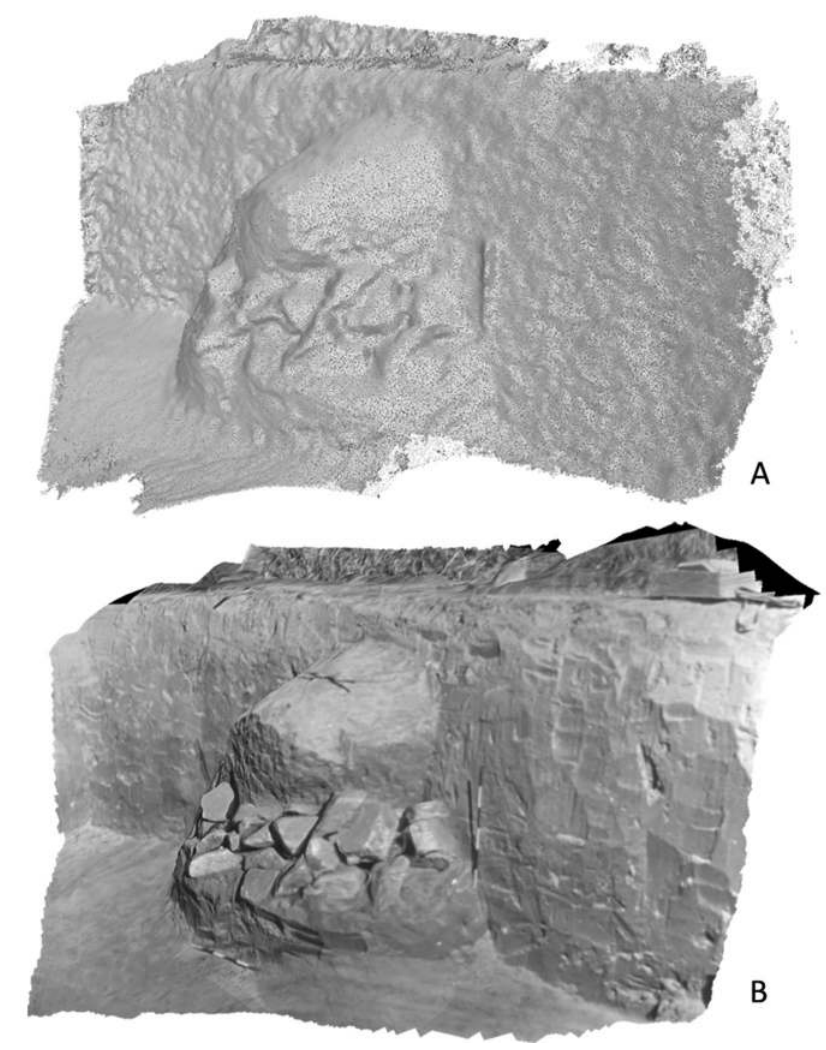

Figure 8 : Restitution photo-grammétrique de l'une des phases de la fouille de la tombe A du tumulus de Kervingar. Nuage dense de points (Dense point cloud) obtenu après alignement des différentes images (A), et visualisation photo-réaliste du modèle (B). Réalisés par E. López-Romero à partir du film des travaux. Archives du Centre de Documentation de l'UMR 6566 CReAAH.

Figure 8: Photogrammetric model of the excavation process in Grave A. Dense point cloud (A) obtained after camera alignment and photomosaic (B) of the visible structures. Obtained from the original film.

tention de modèles. De même, le niveau de pierres éparses trouvé dans le quadrant sud-ouest de la fouille (fig. 9; Giot, inédit a, p. 6-7) n'a malheureusement pas pu être reconstitué : le mouvement de la caméra est trop rapide et a été réalisé à partir de deux points pivotants, l'un depuis le sud et l'autre depuis le nord de la fouille. Nous nous attendions à une meilleure qualité du modèle obtenu pour pouvoir procéder à une analyse de détail de la structure, dont il n'existe, parmi les documents conservés, qu'un plan schématique inutilisable, ce qui aurait peut-être pu contribuer à son interprétation.

Il est donc important de souligner que, aussi bien dans les articles publiés que dans les archives documentaires de la fouille, il existe très peu de documents graphiques et photographiques sur le site. Le plan publié n'offre en outre qu'une vision statique, qui correspond à l'aspect final des travaux et aux structures mises au jour et dégagées de leur contexte. Le court-métrage et les produits dérivés de son analyse numérique nous permettent de compléter ces informations et de nous approcher, de façon inédite, de certaines caractéristiques du site et des travaux.

Les procédures de dégagement et d'ouverture des chambres paraissent spécialement intéressantes à cet égard. Largement conditionnées par la méthode de fouille (pontstémoins), leur analyse structurale et l'accès à leur intérieur se voient limités du fait de leur insertion dans les différentes coupes stratigraphiques. Faute d'une autre alternative, les murets des parois des chambres durent être partiellement démantelés là où ils étaient exposés pour accéder à l'espace funéraire.

Dans le cas de la «tombe accessoire » A, dans le quadrant nord-est de la fouille, on avait initialement isolé une couche sédimentaire sous laquelle, à environ $0,75 \mathrm{~m}$ du point le plus élevé de celle-ci, apparaissaient les premières pierres qui dessinaient une structure en forme semi-circulaire (fig. 8). Cet ensemble s'organise autour d'une dalle de plus grandes dimensions, dont on ne put apprécier initialement que l'extrémité ouest. Une fois les sédiments retirés, le sommet de la dalle apparut et les pierres du muret latéral furent retirées progressivement; parmi elles, une grande dalle allongée (environ 1,25 $\mathrm{m}$ de longueur pour 0,35 $\mathrm{m}$ de largeur maximale), dont le rôle architectonique devait être très important, était disposée en forme de linteau au sommet de la paroi ouest de la chambre. La reconstruction tridimension-

\begin{tabular}{|c|c|c|c|c|c|c|}
\hline structure & situation & description & $\begin{array}{c}\text { n. d'images } \\
\text { utilisees }\end{array}$ & $\begin{array}{c}\text { n. d'images cor- } \\
\text { rectement alignees }\end{array}$ & $\begin{array}{c}\text { n. de faces modele } \\
3 \mathrm{~d}\end{array}$ & $\begin{array}{c}\text { filter steps } \\
\text { (depth smooth) }\end{array}$ \\
\hline tombe a & quadrant ne & $\begin{array}{c}\text { dégagement du } \\
\text { niveau de pierres } \\
\text { du cairn }\end{array}$ & 49 & 49 & 820000 \\
\hline tombe a & quadrant ne & $\begin{array}{c}\text { ouverture de la } \\
\text { chambre }\end{array}$ & 21 & 21 & 580000 \\
\hline tombe c & quadrant sw & $\begin{array}{c}\text { vue extérieure de } \\
\text { la tombe c }\end{array}$ & 29 & 25 & $1.17 \mathrm{~m}$ & 25 \\
\hline
\end{tabular}

Tableau 1 : Description et caractéristiques des modèles obtenus. 


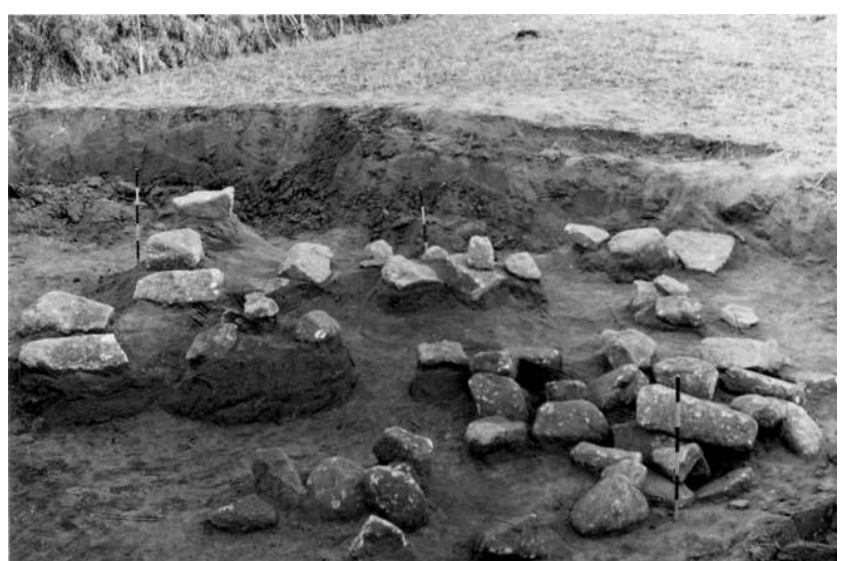

Figure 9 : Niveau de pierres localisé dans le quadrant S-O de la fouille du tumulus de Kervingar. Cliché P.-R. Giot. Archives du Centre de Documentation de l'UMR 6566 CReAAH.

Figure 9: Scattered stones in the southwest quadrant of the excavation.

nelle de ce moment de la fouille nous a permis d'identifier des traces d'extraction ou de régularisation du bloc, traces totalement invisibles aussi bien dans l'enregistrement que dans les quelques photographies conservées (fig. 10).

Dans le secteur sud-ouest, dans l'angle formé par les ponts-témoins ouest et sud, le flanc sud-ouest du cairn central du monument apparut; cette structure fut également démantelée pour dégager la dalle de couverture de la grande " tombe centrale» B. Comme dans le cas de la chambre A, l'espace intérieur n'était pas comblé et fut directement atteint pour récupérer les objets déposés à l'intérieur; il n’y a pas d'images sur l'échantillonnage des restes de bois du plancher de la chambre. Comme nous l'avons signalé plus haut, ces structures n'ont pas pu être reconstituées.

Dans ce même secteur, à l'extrême sud de la zone fouillée, fut découverte la "tombe accessoire » C. Le temps de tournage accordé à cette dernière est extrêmement limité, mais avec une certaine diversité des points de vue de la structure, ce qui a permis tout de même d'en obtenir un modèle (fig. 11). Comme dans le cas du bloc allongé de la " tombe " $\mathrm{A}$, de possibles traces d'extraction, quoique moins nettes, sont visibles sur la dalle de couverture. Un filtrage a été appliqué au modèle sous le logiciel MeshLab (C) (Depth Smooth filtering) pour améliorer sa visualisation. Deux ensembles de pierres situés devant la chambre, et visibles uniquement pendant quelques instants du tournage, semblent disposés circulairement; l'absence de toute représentation de telles structures dans l'ensemble des plans aussi bien publiés qu'inédits nous fait penser à un regroupement de blocs dégagés par l'équipe au cours de la fouille.

À l'exception d'une figuration schématique de la position couchée du vase à quatre anses de la " tombe centrale " B dans une des publications (Giot, 1954), aucun croquis ou
Figure 10 : Vue sommitale du modèle photo-grammétrique de la tombe $\mathrm{A}$, avec indication des traces d'extraction du bloc allongé, et photogramme du film montrant un instant du dégagement de la structure. Archives du Centre de Documentation de l'UMR 6566 CReAAH.

Figure 10: Upper view of the photogrammetric model for Grave A, showing the presence of extraction marks in the elongated stone. In the upper left-hand corner is a still image from the film of the excavation of the chamber.

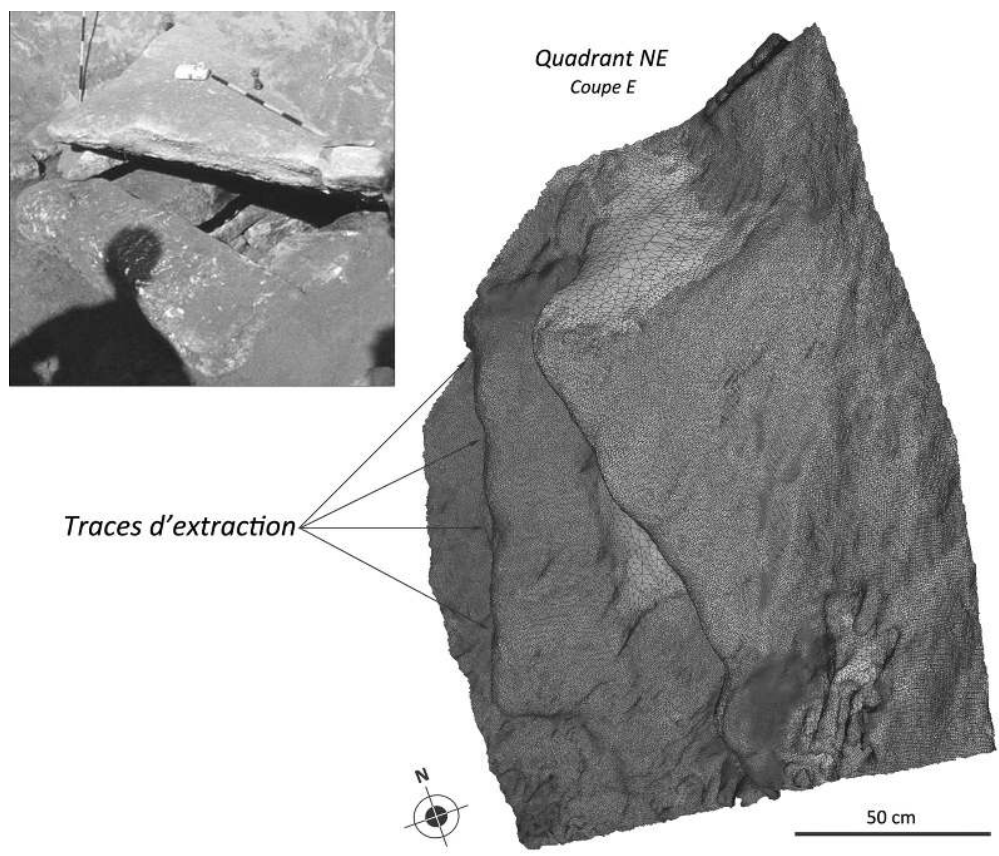




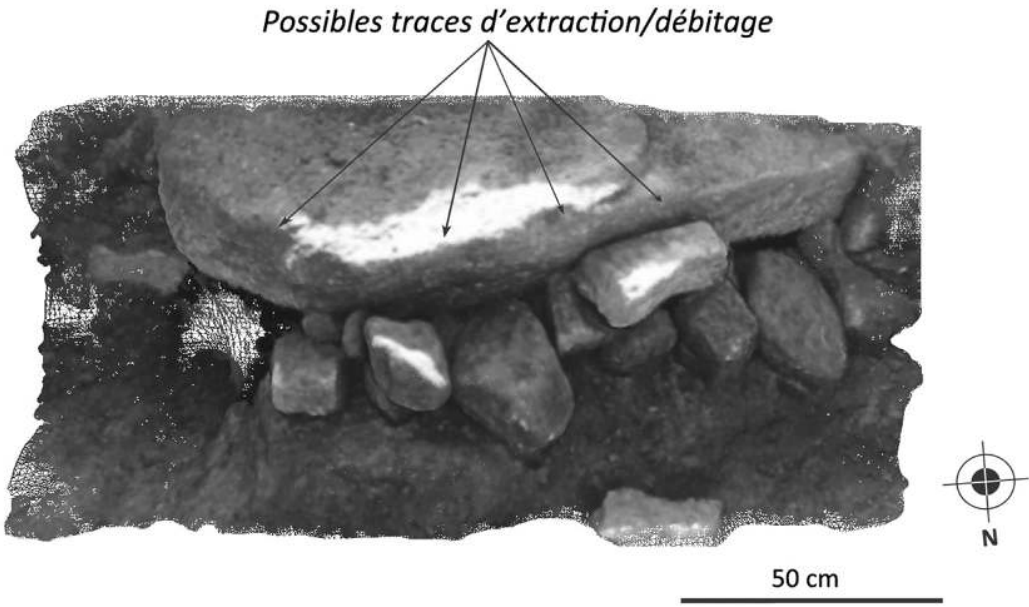

Figure 11 : Reconstruction photo-grammétrique de la partie nord de la Tombe $\mathrm{C}$ du tumulus de Kervingar. Réalisée par E. López-Romero à partir du film des travaux. Archives du Centre de Documentation de l'UMR 6566 CReAAH.

Figure 11: Photogrammetric model of the northern end of Tombe C. Obtained from the original film.

plan de la situation des objets à l'intérieur des chambres ne semble avoir été fait. Des indications centimétriques de leur position par rapport aux parois intérieures sont cependant renseignées dans le cahier de la fouille, et nous avons pu également retrouver deux photographies inédites montrant les vases des chambres A (fig. 12) et B (non reproduite ici à cause de sa mauvaise qualité) au moment de leur ouverture. Par contre, le film rend compte du moment de l'extraction du contenu de ces deux tombes. Pour la petite histoire, la façon dont les vases complets, tout juste sortis de leur contexte de déposition, sont exposés par Louis L'Hostis devant la caméra manifeste une vive émotion spontanée face à l'objet archéologique.

\section{Discussion}

Indépendamment ici des discussions sur les contextes chronologique et culturel du tumulus de Kervingar, le retour à la fouille de ce monument à partir de toute une série d'éléments inédits nous a permis de revenir sur l'une des étapes clés de la formation de l'archéologie comme discipline scientifique officielle en France.

À cette époque, et surtout dès la fin du conflit mondial, P.-R. Giot entame une série d'actions (seul ou en équipe) qui vont le conduire au développement progressif d'une démarche scientifique précise. Cette démarche sera celle qui posera les prémices du laboratoire d'anthropologie, préhistoire, protohistoire et Quaternaire armoricains et qui marquera, par son évolution, le caractère de la recherche d'une grande partie de l'Ouest de la France jusqu'à la fin du $\mathrm{xx}^{\mathrm{e}}$ siècle. Il s'agit là d'une recherche pluridisciplinaire qui couvrira non seulement le champ de l'archéologie (au sens large) mais également ceux de l'histoire, de la géologie, la paléobiologie, l'anthropologie physique, la chimie...

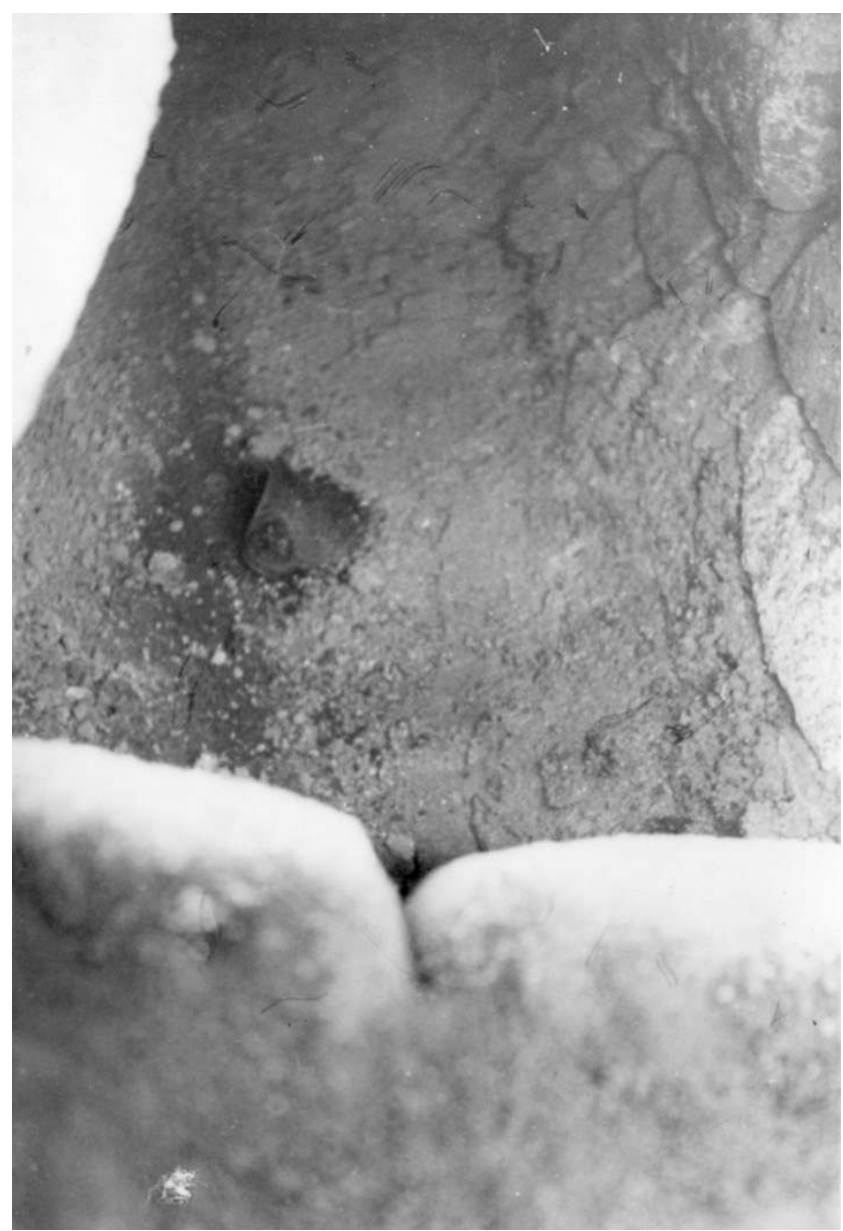

Figure 12 : Localisation in situ du vase à deux anses de la tombe A au moment d'ouverture de la chambre. Cliché P.-R. Giot, Archives du Centre de Documentation de l'UMR 6566 CReAAH.

Figure 12: A still image from the film of the opening of grave A showing the position of the two handled vase found. 
Par ses origines liées à l'institut finistérien d'études préhistoriques et par sa titularisation professionnelle dès 1943, Giot incarne le passage d'une archéologie de l'avant-guerre (celle de l'initiative individuelle, souvent bâtie autour des sociétés savantes) vers une archéologie officielle, science moderne et pluridisciplinaire. La focalisation quasi exclusive de son travail sur le contexte armoricain, ainsi que l'absence d'une chaire de protohistoire à l'Université ont pu jouer d'après certains auteurs (Audouze, 2003; note 20) contre une plus grande extension de l'influence de ses idées à l'échelle nationale et contre une meilleure reconnaissance de son travail en France au même niveau que les F. Bordes, G. Camps ou A. Leroi-Gourhan, responsables de la création des autres grands laboratoires de référence instaurés dans les années 1960 (Audouze, 2003). Mais Giot restait fidèle à l'idée d'une décentralisation de la recherche et ne manquait pas l'occasion de souligner ses convictions, même lorsqu'il prenait la responsabilité de postes de premier rang au sein des instances parisiennes les plus renommées : "Établi en province sans doute, sans être un provincial, mais en tout cas champion de la décentralisation, ou si l'on veut, de la déconcentration. [...] notre matière même est liée aux terroirs, aux pays, aux sols, aux sous-sols, c'est essentiellement une science régionale, et il s'ensuit que toute structuration administrative et scientifique qui lui convienne doit être régionalisée " (Giot, 1961, p. 9; voir une critique dans Gouletquer, 2009, p. 466). Et pourtant, la reconnaissance et l'impact de ses idées et de celles de son équipe à l'échelle internationale sont indiscutables, fait sans doute favorisé par sa profonde connaissance du monde anglo-saxon et par son bilinguisme ${ }^{5}$.

Même si la configuration de cette démarche scientifique a été, comme nous l'avons signalé, progressive, la fouille de Kervingar nous apparaît comme un point d'inflexion dans sa mise en pratique. Malgré certains problèmes en ce qui concerne la réalisation (par exemple, limitations du système des ponts-témoins pour la correcte compréhension des structures et pour l'accès à l'espace intérieur des tombes) ou la publication (par exemple, pauvre qualité du plan général de la fouille, coupe et dessins restés inédits) des travaux, le saut qualitatif de cette démarche par rapport au contexte régional de l'époque réside dans la planification et dans la systématisation. Planification du travail avant, pendant et après la fouille, avec développement d'une perspective critique (nécessité d'effectuer des fouilles intégrales, présentation du site archéologique comme une entité structurellement complexe et diachronique). Systématisation des méthodes mises en œuvre; perspective d'exploitation des

5. Pierre-Roland Giot était le fils d'Henri Giot et d'Annie Louise Baxter, cette dernière de nationalité anglaise. résultats adaptée aux innovations techniques du moment et à leur futur potentiel d'évolution.

Kervingar représente également le point de départ de ce qui sera l'une des lignes de recherche les plus fructueuses du laboratoire d'anthropologie, celle de l'étude du monumentalisme funéraire du Néolithique et de l'âge du Bronze dans l'Ouest de la France. L'incorporation, dès cette même année 1953, de collaborateurs tels que Jacques Briard (19332002), Jean L'Helgouac'h (1933-2000) ou encore Yves Coppens (dont quelques-uns des premiers travaux sont liés au Laboratoire en qualité d'étudiant à la faculté de sciences de Rennes) servira à la consolider et à la diversifier. Cette ligne viendra très tôt se profiler davantage par la précipitation des événements. Les découvertes de sites et de monuments se succèdent. C'est le moment du début des travaux à l'île Carn (1954), à Barnenez (1955) puis à l'île Guennoc (1960). Architectures massives, multi-phases et complexes pour l'étude desquelles l'expérience de Kervingar (avec séquençage relatif des trois chambres funéraires et de leurs cairns puis condamnation finale par un grand tumulus) a sans doute joué son rôle. Dans leur cas, il est cependant nécessaire de repenser les méthodes d'action, les mesures à prendre en matière de protection, consolidation et restauration (Giot, 1987). C'est également pour l'équipe, selon les mots de Jacques Briard, le moment des "Robinsons archéologues" (Briard, 2000, p. 332), ceux de la redécouverte d'une archéologie du littoral riche, mais parfois réalisée dans des conditions très difficiles.

Même si, sur le plan chronologique et interprétatif, les travaux de Jacques Briard (1984), de Jean L'Helgouac'h (1965) et de P.-R. Giot lui-même (1987) viennent bientôt modifier le cadre chrono-typologique, les catégories et les interprétations établies pendant les années 1950 dans la péninsule armoricaine (et, par leur implication, dans beaucoup d'autres régions de la façade atlantique de l'Europe), le renouveau théorique et méthodologique alors implanté conservera à plusieurs titres son caractère pionnier.

Document oublié, sauvé in extremis de la destruction, le film sur la fouille de Kervingar nous renseigne, dans une tout autre perspective, sur ce renouveau. Au-delà de sa valeur intrinsèque comme document visuel de l'époque, l'utilisation des images contenues dans l'enregistrement pour restituer certains éléments de la fouille nous oriente vers une nouvelle forme d'exploitation scientifique des archives anciennes.

Cette restitution tridimensionnelle par photogrammétrie permet d'obtenir de nouvelles données quantitatives (possibilité de mesurer, calculer des volumes, etc.) et qualitatives (identification d'éléments inédits) qui autrement seraient irrécupérables. 
De fait, nous avons notamment pu reconstituer différentes phases des travaux (tombe A) et identifier des éléments architecturaux et technologiques inédits (traces d'extraction, débitage ou mise en forme des blocs). Ce type d'analyse se présente alors comme un outil essentiel pour la validation de certaines hypothèses archéologiques sur l'espace bâti et sur l'architecture de sites détruits ou devenus inaccessibles, validation jusqu'ici limitée par le caractère bidimensionnel de la documentation conservée (Large, 2013).

Il faut cependant souligner que cette perspective est largement conditionnée par les caractéristiques mêmes des archives. Puisque les images n'ont pas, à l'origine, été prévues pour de telles reconstitutions, les principaux problèmes que nous avons rencontrés pour une correcte modélisation concernent :

- le faible nombre d'images généralement disponible pour l'objet étudié;

- l'absence d'une diversité de perspectives de l'objet (déficit d'images présentant l'objet sous différents angles);

- l'éclairage peu approprié du sujet (les zones dans l'ombre ne pourront pas être restituées);

- l'absence d'échelle graphique pour certains objets ou éléments;

- l'obligation de travailler avec des systèmes de coordonnées relatives;

- l'absence d'informations sur l'appareil (pour atteindre une meilleure qualité dans la reconstruction et permettre le calcul des paramètres internes) et sur la calibration des images (focale, obturateur, paramètres ISO...);

- la difficulté d'évaluer, faute de points de contrôle, la précision des modèles obtenus.

Nos expériences menées avec le film de Kervingar et avec d'autres fonds d'images datant du début du Xx siècle (LópezRomero, 2014) nous poussent, en tout cas, à être très optimistes sur le potentiel de cette approche. Paradoxalement, le futur le plus prometteur des nouvelles méthodes d'analyse numérique en archéologie réside peut-être dans un retour aux plus vieilles archives de la discipline.

\section{Remerciements}

Ce travail a été réalisé dans le cadre des projets ICARE (Iconographie et Collections d'Anthropologie de Rennes, CReAAH UMR6566) et eSCOPES (Evolving spaces: coastal landscapes of the Neolithic in the European Lands Ends, Marie Curie-IEF 2013-2015, Durham University). Les travaux de numérisation des films ont été réalisés en 2011 par l'agence Génération Vidéo [http://www.generation-video.fr/], et son financement a été assuré par l'ATLA (Association des Travaux du Laboratoire d'Anthropologie de Rennes). Les photos, dessins, plans et coupes inédits du site, préservés dans les archives de l'UMR6566 CReAAH-Université de Rennesl, ont été numérisés par Loüc Langouët (AMARAI). Merci à Catherine Le Gall (Centre de Documentation, CReAAH UMR6566), pour son aide constante, à $M$. Norbert L'Hostis /http://genealhostis.pagesperso-orange.fr/index.html], pour ses renseignements concernant le Dr. vétérinaire Louis L'Hostis, et à Kurt Gron (Durham University), pour la relecture du résumé en anglais. Merci également à Charles-Tanguy Le Roux et à un autre rapporteur anonyme pour leurs commentaires, corrections et précisions, qui ont contribué à améliorer ce texte.

\section{Bibliographie}

Agarwal S., Furukawa Y., Snavely N., Simon I., Curless B., Seitz S.M. et Szeliski R., 20 I I - "Building Rome in a Day ", Communications of the ACM, 54, 10, p. 105-112. doi :10.1145/2001269.2001293.

Audouze F., 2003 - "La préhistoire et le CNRS ", La revue pour l'histoire du CNRS, 8 [http://histoire-cnrs.revues.org/551\#text], consulté le 9 août 2013.

Beraldin J.-A., Blais F., Boulanger P., Cournoyer L., Domey J., El-Hakim S.F., Godin G., Rioux M. et Taylor J., 2000 - "Real world modelling through high resolution digital 3D imaging of objects and structures ", ISPRS Journal of Photogrammetry and Remote Sensing, 55 (4), p. 230-250.

Briard J., I984 - Les tumulus d'Armorique, Paris, Picard (L'âge du Bronze en France, 3).

Briard J., 2000 - « Jean L'Helgouac'h (1933-2000) », Bulletin de la Société Préhistorique Française, 97 (2), p. 331-333.

Briard J., Gomez De Soto J., Milcent P.-Y. et Pautreau J.-P., 200 I - «Les recherches sur l'âge du Bronze en PoitouCharentes, Centre, Pays-de-la-Loire, Bretagne et BasseNormandie ", Documents d'Archéologie Méridionale, 24, p. 259-266.

Butler J. J. et Waterbolk H. T., I974 - «La fouille de A. E. van Giffen à "La Motta”. Un tumulus de I'âge du Bronze ancien à Lannion (Bretagne) ", Palaeohistoria, 16, p. 107-167.

Cogné et Giot P.-R., I95 I - "L'âge du Bronze ancien en Bretagne ", L'Anthropologie, 55, p. 425-444.

Collectif, 2007 - «Marthe et Saint-Just Péquart, archéologues des îles. De Houat à Hoëdic, 1923-1934 ", Melvan, la revue des deux îles, numéro spécial, 4, $285 \mathrm{p}$.

Daire M.-Y. et López-Romero E., 20 i I - Au fil de l'eau... Le littoral et son étude à travers les archives de l'ancien laboratoire d'anthropologie de Rennes, Livret de l'exposition Rennes, CReAAH UMR6566, 26 p.

Daire M.-Y. et López-Romero E., 20 I 2 - " Le projet ICARE et l'exposition "Au fil de l'eau..." ", Journée Archéologie, Archéosciences, Histoire, Rennes 24/03/2012, Université de Rennes 1, p. 35-36. 
Daire M.-Y., López-Romero E. et Le Gall C., 20I3 - «Théodore Monod (1902-2000) et l'archéologie bretonne - Note sur un épisode méconnu de la vie du "fou du désert », Revue archéologique de l'Ouest, 30, p. 289-301.

Damour A., I 864 - «Sur la Callaïs, nouveau phosphate d'alumine hydraté recueilli dans un tombeau celtique du Morbihan ", Comptes rendus de l'académie des sciences, 59, p. 936-939.

Doneus M., Verhoeven G.J.J., Fera M., Briese C., Kucera M. et Neubauer W., 20 I I - «From deposit to point cloud. A study of low-cost computer vision approaches for the straightforward documentation of archaeological excavations ", Geoinformatics, 6 (XXIII $^{\text {rd }}$ International CIPA Symposium), p. 81-88.

Gabillot M., 20io - "Neue Forschungen zu den Frühbronzezeitlichen " armorikanischen Tumuli ", Tagungen des landesmuseum für vorgeschichte halle. Der Griff nach den Sternen: Internationales Symposium in Halle (Saale) 16-21 Februar 2005, p. 207-215.

Gillings M., 2000 - «Plans, elevations and virtual worlds: the development of techniques for the routine constructions of hyperreal simulations ", in Barceló J., Forte et SAnders H. (éd.), Virtual reality in Archaeology-Computer Applications and Quantitative Methods in Archaeology (CAA), Oxford, British Archaeological Reports International Series, 843, p. 59-69.

Giot P.-R., I 947 - "Le tumulus mégalithique de Beg-anDorchenn ", Gallia, 5, p. 167-170.

Giot P.-R., I 948 - « L'organisation des recherches paléo-ethnologiques ", in Cossira H. et Colin A. (dir.), À travers les départements meurtris - Le Finistère, éd. La France au combat, 239 p.

Giot P.-R., I95 I - Armoricains et bretons, étude anthropologique, thèse de sciences, Rennes, Travaux de l'Institut d'anthropologie générale de la faculté des sciences de Rennes, Imprimerie Les Nouvelles de Bretagne, 160 p., 36 fig.

Giот P.-R., I953a - "Quelques tombes finistériennes de l'âge du Bronze découvertes fortuitement ", Bulletin de la société archéologique du Finistère, 79, p. 31-42.

Giot P.-R., I953b - «De l'intérêt de la réouverture des tumulus fouillés antérieurement ", Bulletin de la société archéologique du Finistère, 79, p. 43-51.

Giот P.-R., I954 - « Le tumulus de Kervingar à Plouarzel, fouilles de septembre 1953 ", Bulletin de la société archéologique du Finistère, 80, p. 111-134.

Gioт P.-R., I956 - «L'application des techniques modernes à la fouille des tumulus des âges du Bronze ancien et moyen de Bretagne ", Bulletin de la société préhistorique de France, 53 (1-2), p. 101-102.

Giot P.-R., I958 - "Glanes préhistoriques et protohistoriques finistériennes ", Bulletin de la société archéologique du Finistère, 84, p. 198-206.

Gioт P.-R., I960 - "Vers une échelle chronologique "absolue" pour la préhistoire et la protohistoire armoricaines, Annales de Bretagne, 67 (1), p. 33-44.
Giot P.-R., I96I - «Vie de la société - discours de P.-R. Giot, président entrant ", Bulletin de la société préhistorique française, 58 (1-2), p. 3-14.

Giot P.-R., I 967 - « Chronique des datations radiocarbones armoricaines ", Annales de Bretagne, 74 (1), p. 150-153.

Giot P.-R., I987 - Barnenez, Carn, Guennoc, Travaux du laboratoire anthropologie-préhistoire-protohistoire armoricaines, Rennes, 2 vol.

Giот P.-R., inédit a - Kervingar, cahier de fouilles, 10-26 septembre 1953, archives du centre de documentation du laboratoire archéosciences UMR 6566 CReAAH - Centre de recherche en archéologie, archéosciences, histoire, Rennes, $14 \mathrm{p}$.

Giот P.-R., inédit b - Tumulus de Kervingar à Plouarzel, fouilles de septembre 1953, notes dactylographiées, archives du centre de documentation du laboratoire archéosciences UMR 6566 CReAAH - Centre de recherche en archéologie, archéosciences, histoire, Rennes, 9 p.

Giot P.-R. et Cogné, J., I 948 - "Fouille d'un tumulus de l'âge du Bronze au bourg de Kersaint-Plabennec ", Bulletin de la Société archéologique du Finistère, 74, p. 80-85.

Giot P.-R. et Guilcher A., I947 - «Essai de chronologie des successions pré- et protohistoriques de la baie d'Audierne (Finistère) ", Bulletin de la Société préhistorique de France, 44 (3-4), p. 116-121.

Gouletquer P., 2009 - "Éteignez bien les braises ", in Cassen S. (dir.), Autour de la Table. Explorations archéologiques et discours savants sur des architectures néolithiques à Locmariaquer, Morbihan (Table des Marchands et Grand Menhir), Laboratoire de recherches archéologiques, CNRS et université de Nantes, p. 463-471.

Grimes W.F., I938 - "A Barrow on Breach Farm, Llanbleddian, Glamorgan ", Proceedings of the Prehistoric Society, 4 (1), p. 107-121.

Grün A., Remondino F. et Zhang L., 2004 - « Photogrammetric Reconstruction of the Great Buddha of Bamiyan, Afghanistan ", The Photogrammetric Record, 19(107), p. 177199.

Hurel A., 2007 - La France préhistorienne de 1789 à 1941, Paris, CNRS Éditions.

IoAnnidis C., Potsiou C. et Badekas J., I996 - «3D Detailed Reconstruction of a demolished Building by using old photographs ", International Archives of Photogrammetry and Remote Sensing, 31 (part B5), p. 16-21.

Laplace-Jauretche G., I95 I - « Le dolmen du Turoun Bouchous, Haute Vallée d'Ossau ", Eusko-Jakintza, 5, p. 257-262.

Laplace-Jauretche G. et Méroc L., I954 - "Application des coordonnées cartésiennes à la fouille d'un gisement ", Bulletin de la Société préhistorique de France, 51 (1-2), p. 58-66.

LARGE J.-M., 20I 3 - "Un nouveau critère de continuité entre le Mésolithique et le Néolithique en Bretagne méridionale ", in 
Guyodo J.-N. et Mens E. (éd.), Les premières architectures en pierre en Europe occidentale. $D u V^{e}$ au $I I^{e}$ millénaire avant J.-C., Rennes, PUR, p.55-77.

Le Pontois L., I 890 - "Exploration du tumulus de Cruguel, commune de Guidel (Morbihan)", Revue archéologique, 16, p. 304-338.

L'Helgouac'H J., I965 - Les sépultures mégalithiques en Armorique, Travaux du Laboratoire d'Anthropologie de la Faculté des Sciences de Rennes, Université de Rennes, 330 p.

López-Romero E., 20I4 - "Out of the box: exploring the 3D modelling potential of ancient image archives, Virtual Archaeology Review, 5(10), p. 107-116 [http://varjournal.es/ doclvarj05_010_11.pdf].

López-Romero E. et Daire M-Y., 20I3 - « The ICARE Project: Insights into the Formation and Consolidation of Archaeology in Western France (ca. 1850-1990) ", Bulletin of the History of Archaeology, 23(1) paper 3, DOI: [http://dx.doi.org/10.5334/ bha.2313].

López-Romero E., Mañana-Borrazás P., Daire M-Y. et GÜImilFARIÑA A., 2OI 4 - " The eSCOPES Project: preservation by record and monitoring at-risk coastal archaeological sites on the European Atlantic façade ", Antiquity Project Gallery, 88 (339), [http://journal.antiquity.ac.uklantplus/projgall].

Milon Y. et Giot P.-R., I954 - " IV Circonscription ", Gallia, 12 (2), p. 401-408

Nicolas C., Pailler Y., Stephan P. et Gandois H., 2013 - « Les reliques de Lothéa (Quimperlé, Finistère) : une tombe aux connexions atlantiques entre Campaniforme et âge du Bronze ancien ", Gallia préhistoire, 55, p. 181-227.

Rocher C., 2013 - Pierre-Roland Giot ou les mutations de la recherche d'après-guerre, Mémoire de master 1, université de Rennes 2 - UFR sciences humaines, master 1 histoire et archéologie, $81 \mathrm{p}$.

Simpson A., Clogg P., Diaz-Andreu M. et Larkman B., 2004 "Towards three-dimensional non-invasive recording of incised rock art ", Antiquity, 78 (301), p. 692-698.

Verhoeven G., 20I I - "Taking computer vision aloft Archaeological three-dimensional reconstructions from aerial photographs with PhotoScan ", Archaeological Prospection, 18, p. 67-73. DOI: 10.1002/arp.399.

Vogel J.-C. et Waterbolk H.T., I963 - « Groningen radiocarbon dates IV ", Radiocarbon, 5, p. 163-202.

Zusammenfassung: Der Grabhïgel von Kervingar (Plouarzel, Finistère): Neue Betrachtungen zu den Anfängen archäologischer Forschung in der Nachkriegszeit in der Bretagne - Ausgehend von einem Konvolut bisher unpublizierten Materials hat dieser Beitrag zum Ziel, die im Jahre 1953 von Pierre-Roland Giot (1919-2002) durchgeführte Ausgrabung des bronzezeitlichen Grabhügels von Kervingar (Plouarzel, Finistère, Frankreich) neu aufzugreifen. Die kritische Durchsicht und Analyse dieser Dokumente und die Diskussion des Forschungskontextes jener Epoche gestatten uns, diese Ausgrabung als eine entscheidende Etappe jener wissenschaftlichen Erneuerung zu charakterisieren, die die französische Archäologie am Ende der 1940 er Jahre erfasste. Insbesondere handelt es sich dabei um eine derjenigen Grundlagenforschungen, die es gestatten, das wissenschaftliche Denken jener Zeit zu verstehen, das schließlich zur Gründung des „Laboratoire d'Anthropologie, Préhistoire, Protohistoire et Quaternaire armoricains" führte und das in der Folge einen großen Teil der archäologischen Forschung im Westen Frankreichs während der zweiten Hälfte des 20. Jahrhunderts prägte. Die Existenz eines bisher unveröffentlichten Films zu den Ausgrabungsarbeiten ermöglichte außerdem, eine neuartige Methode der Bildanalyse anzuwenden, bei der ein dreidimensionales digitales Model eines Teils der Ausgrabung erstellt werden konnte. Dieses Vorgehen ist ein gutes Beispiel dafür, neue Wege bei der wissenschaftlichen Auswertung von Altgrabungen und dem vorliegenden Archivmaterial zu beschreiten.

Resumen: El túmulo Keringar (Plouarzel, Finistère): una nueva mirada en los inicios de la investigación arqueológica en el momento de la posguerra en Bretaña - A partir de una serie de documentos inéditos, este trabajo trata de la excavación de 1953 del túmulo de la Edad de Bronce temprana de Kervingar (Plouarzel, Finistère) por Pierre-Roland Giot (1919-2002). La revisión de estos documentos y la discusión sobre el contexto de la época nos permiten proponer esta excavación como una de las piezas clave de la renovación científica que tendrá lugar en la arqueología francesa a partir de la década de 1940. Esta excavación constituye, ante todo, un trabajo fundamental para entender el pensamiento cientifico que dará lugar a la creación del "Laboratorio de Antropología, Prehistoria, Protohistoria y Cuaternario armoricanos" y, finalmente, que marcará gran parte de la investigación arqueológica en el oeste de Francia a lo largo de la segunda mitad del siglo XX. La existencia de una película inédita sobre este trabajo también nos ofrece la oportunidad de proponer una metodología innovadora para el análisis de imágenes con la reproducción tridimensional digital de una parte de la excavación. Esta perspectiva abre nuevas oportunidades para la explotación cientifica de los archivos antiguos.

Schlüsselwörter: Grabhügel, ältere Bronzezeit, Geschichte der Archäologie, Photogrammetrie, Structure from Motion (SfM), Bildbearbeitung, Bretagne, Finistère.

Palabras clave: túmulo, Edad del Bronce antiguo, historia de arqueología, fotogrametría, Estructura del Movimiento (SFM), procesamiento de imágenes, Bretaña, Finistère. 